\title{
Vibration Based Damage Detection of Rotating Beams
}

\author{
Stanislav Stoykov ${ }^{1, *}$, Emil Manoach ${ }^{2}$ and Maosen Cao ${ }^{3}$ \\ ${ }^{1}$ Institute of Information and Communication Technologies, Bulgarian Academy of Sciences, Bulgaria \\ ${ }^{2}$ Institute of Mechanics, Bulgarian Academy of Sciences, Bulgaria \\ ${ }^{3}$ Department of Engineering Mechanics, Hohai University, Nanjing, China
}

\begin{abstract}
The early detection and localization of damages is essential for operation, maintenance and cost of the structures. Because the frequency of vibration cannot be controlled in real-life structures, the methods for damage detection should work for wide range of frequencies. In the current work, the equation of motion of rotating beam is derived and presented and the damage is modelled by reduced thickness. Vibration based methods which use Poincaré maps are implemented for damage localization. It is shown that for clamped-free boundary conditions these methods are not always reliable and their success depends on the excitation frequency. The shapes of vibration of damaged and undamaged beams are shown and it is concluded that appropriate selection criteria should be defined for successful detection and localization of damages.
\end{abstract}

\section{Introduction}

Modelling of the dynamic behavior of rotating beams is very important for many engineering applications like wind power generators, helicopter blades, etc. The early detection and localization of damages of these structures is essential for their operation and maintenance. Vibration based methods for damage detection are among the most popular methods for health monitoring of structures. The easiest ones are based on the modal properties of the structures. The lowest natural frequencies, however, are not very sensitive to small damages and they are not indicative to predict the presence of damage and even less its location. The modes of vibrations are more sensitive to damages. The difficulty for their use comes from the fact that many sensors have to be used. This difficult is overcame by using the scanning laser Doppler vibrometer (SLDV) techniques. Even by using SLDV the modal based features could be difficult to extract when the objects of monitoring are large and/or complex structures and when some parts of these structures are either inaccessible or very difficult for taking measurements. In the last decades the vibration based methods for damage detection are not limited to monitoring the changes in modal properties of the structures but are extended to analysis of the response of the structures in the time domain. The researches using such methods try to extract some features from the signals in the forced response of structures. These methods are more appropriate for in-operation structural health monitoring (SHM) of structures subjected to dynamic loading and they poses a generality. A review of the time domain method for SHM with accent on the ones using the nonlinear response of the structure could be found in [1]. A big and very promising group of methods, which use measured time series are the one, used the statistical approaches [1-3]. One of the time-series methods is the method based on the Poincaré maps of the forced response of the structures $[3,4]$. It was tested and verified experimentally for composite beams with delamination in [5]. This approach was not tested, however, for the important from the practical point of view case for rotating beam. In the present work the Poincaré map based method for damage assessment was tested for rotating beams and conclusions about its applicability and further development was drawn.

\section{Mathematical model}

It is assumed that the beam can vibrate in longitudinal and transverse directions and rotate in a plane perpendicular to the plane of transverse vibrations. The Timoshenko's beam theory is considered and the rotation of the beam is introduced into the equation of motion through the inertia forces. Geometrical type of nonlinearity is included in the model. The equation of motion is derived by the principle of virtual work and it is discretized by the finite element method.

Considering Timoshenko's beam hypothesis, the displacements in longitudinal and transverse directions are expressed by the displacements on the middle line and the rotation of the cross section:

$$
\begin{aligned}
& u(x, y, z, t)=u_{0}(x, t)+z \phi_{y}(x, t), \\
& w(x, y, z, t)=w_{0}(x, t),
\end{aligned}
$$

\footnotetext{
*Corresponding author: stoykov@parallel.bas.bg
} 
where $u_{0}(x, t)$ and $w_{0}(x, t)$ represent the displacements in longitudinal and transverse directions and $\phi_{y}(x, t)$ represent the rotations of the cross section about $y$ axis.

Nonlinear strain-displacement relations are considered in the model, which are known as geometrical type of nonlinearity. Hence the strains are expressed as:

$\varepsilon_{x}=\frac{\partial u}{\partial x}+\frac{1}{2}\left(\frac{\partial w}{\partial x}\right)^{2}$

$\gamma_{x z}=\frac{\partial w}{\partial x}+\frac{\partial u}{\partial z}+\frac{\partial w}{\partial x} \frac{\partial w}{\partial z}$.

The material of the beam is assumed to be homogeneous and isotropic. The following constitutive relations are used:

$\left\{\begin{array}{c}\sigma_{x} \\ \tau_{x z}\end{array}\right\}=\left[\begin{array}{cc}E & 0 \\ 0 & \lambda G\end{array}\right]\left\{\begin{array}{c}\varepsilon_{x} \\ \gamma_{x z}\end{array}\right\}$

where $\sigma_{x}$ and $\tau_{x z}$ are bending and shear stresses, $E$ is the Young's modulus of the material, $G$ is the shear modulus and $\lambda$ is shear correction factor.

The rotation of the beam is included in the equation of motion through the inertia forces. For that purpose, two coordinate systems are considered: one fixed, denoted by $S_{0}$, and another one rotating about the fixed coordinate system denoted by $S_{1}$. The displacement fields (1) are written in the rotating coordinate system $S_{1}$, which is assumed to rotate with constant speed $\dot{\vartheta}$ $(\mathrm{rad} / \mathrm{s})$. The absolute acceleration of an arbitrary point $\mathrm{P}(x, y, z)$ from the beam is expressed with respect to the fixed coordinate system $S_{0}[6]$ :

$\boldsymbol{a}_{\mathrm{P}_{\mathrm{S}_{0}}}=\boldsymbol{a}_{\mathrm{P}_{\mathrm{S}_{1}}}+\boldsymbol{\omega} \times(\boldsymbol{\omega} \times \boldsymbol{r})$,

where $\boldsymbol{a}_{\mathrm{P}_{\mathrm{S}_{0}}}$ represents the absolute acceleration of point $\mathrm{P}, \boldsymbol{a}_{\mathrm{P}_{\mathrm{S}_{1}}}$ represents the relative acceleration of point $\mathrm{P}, \omega$ represents the angular velocity vector of the rotating coordinate system, and $\boldsymbol{r}$ represents the position vector of point $\mathrm{P}$ with respect to the origin of $S_{1}$.

The equation of motion is derived by the principle of virtual work:

$\delta W_{V}+\delta W_{i n}+\delta W_{E}=0$

where $\delta W_{V}$ represents the virtual work of internal forces, $\delta W_{i n}$ represents the virtual work of inertia forces, $\delta W_{E}$ represents the virtual work of external forces.

The resulting partial differential equations are discretized by the finite element method. Quadratic elements with three nodes are used for the discretization. The following system of nonlinear ordinary differential equations is obtained:

$\mathbf{M} \ddot{\mathbf{q}}+\beta \mathbf{K}_{\mathbf{l}} \dot{\mathbf{q}}+\mathbf{K}_{\mathbf{l}} \mathbf{q}+\mathbf{K}_{\mathbf{n l}}(\mathbf{q}) \mathbf{q}+\mathbf{T}(\dot{\vartheta}) \mathbf{q}=\mathbf{f}-\mathbf{r}(\dot{\vartheta})$

where $\mathbf{M}$ represents the mass matrix, $\mathbf{K}_{\mathbf{l}}$ represents the stiffness matrix of constant terms, $\mathbf{K}_{\mathbf{n l}}(\mathbf{q})$ represents the stiffness matrix that depends on the vector of generalized coordinates $\mathbf{q}$, it results from the geometrical nonlinearity of the model and introduces quadratic and cubic terms at the equation of motion. $\mathbf{f}$ represents the vector of generalised external forces. $\mathbf{T}(\dot{\vartheta})$ and $\mathbf{r}(\dot{\vartheta})$ are matrix and vector that arise from the rotation of the beam and depend on the speed of rotation. Stiffness proportional damping is introduced in the model.

\section{Damage detection formulas}

The Poincare map based method introduced in $[3,4]$ is based on the differences which arise in the phase space of the responses of the healthy and damaged structures. The Poincaré maps preserves many properties of periodic and quasiperiodic orbits of the system and has a lower-dimensional state space then the time-history series.

The method requires calculation of a damage index constructed in the following way:

$I_{i}^{d}=C\left(S_{i}^{u}-S_{i}^{d}\right)$

where

$$
\begin{aligned}
& S_{i}^{u}=\sum_{j=1}^{N_{p}-1} \sqrt{\left(w_{i, j+1}^{u}-w_{i, j}^{u}\right)^{2}+\left(\dot{w}_{i, j+1}^{u}-\dot{w}_{i, j}^{u}\right)^{2}} \\
& S_{i}^{d}=\sum_{j=1}^{N_{p}-1} \sqrt{\left(w_{i, j+1}^{d}-w_{i, j}^{d}\right)^{2}+\left(\dot{w}_{i, j+1}^{d}-\dot{w}_{i, j}^{d}\right)^{2}} \\
& i=1,2 \ldots N_{\text {nodes }}
\end{aligned}
$$

In Eqs. (7) and (8), $N_{\text {nodes }}$ is the number of nodes, $N_{\mathrm{p}}$ is the number of points on the Poincare map $\left(w_{i j}^{u}, \dot{w}_{i j}^{u}\right)$ and $\left(w_{i j}^{d}, \dot{w}_{i j}^{d}\right)$ denote the $j^{\text {th }}$ point on the

Poincaré maps of the undamaged and the damaged states of $i^{\text {th }}$ node, respectively.

$C$ is a constant which could be connected with the number of the nodes in the Poincare map (for example $C=1 / N_{\mathrm{p}}$.). In our previous studies, $\mathrm{C}$ was chosen to be a function of $x$, as $C=S_{i}^{u}$. In the most cases this approach gives very good results. In some cases, however $S_{i}^{u}$ could be very small at the areas close to the clamped ends of the structure and this could lead to wrong results.

This difference given with Eq. (7) is taken as a measure for the global change (during the full period of vibration) in the dynamic behaviour of the damaged structure comparing with the undamaged one. If the function $I^{D}(x)$ has a maximum and it is strong concave in the vicinity of the maximum it means that the damage is located close to the nodes where is located the maximum. Thus for the structure with damage the nodes close to the maximal value of the function will represent the damaged area.

In [7], by using the analogy of the Poincaré map based method with the modal displacement method and the fact that the modal curvature method gives better results then the modal displacement method, the damage index (eq. (7)) was improved . 
The following damage index based again on the Poincaré maps was suggested there:

$$
D I 2_{i}=C\left(\left|\frac{\partial^{2}\left(S_{i}^{u}-S_{i}^{d}\right)}{\partial x^{2}}\right|\right) i=1,2, \ldots N_{\text {nodes }}
$$

This expression, of course, is far more complicated than the modal curvature damage index but the second derivatives of functions of the displacements and the velocity of the response are included in it.

If one wants to enlarge the differences in the second derivatives of these functions the following formula can be used:

$$
D I 3_{i}=C^{2}\left(\left|\left(\frac{\partial^{2} S_{i}^{u}}{\partial x^{2}}\right)^{2}-\left(\frac{\partial^{2} S_{i}^{d}}{\partial x^{2}}\right)^{2}\right|\right) \quad i=1,2, \ldots, N_{\text {nodes }}
$$

\section{Numerical experiments}

A beam with homogeneous and elastic material is considered for the numerical experiments. The damage is modelled by reducing the thickness of the damaged area of the beam. The material properties of the beam are: Young modulus $E=70 \mathrm{GPa}$, density $\rho=2778 \mathrm{~kg} / \mathrm{m}^{3}$, Poisson ratio $v=0.3$ and the geometrical properties are: length $l=0.58 \mathrm{~m}$, width $b=0.02 \mathrm{~m}, \quad$ thickness $h=$ $0.002 \mathrm{~m}$. The reduced thickness of the damaged beam is assumed to be $h^{d}=0.0015 \mathrm{~m}$. The equation of motion is discretized by quadratic finite elements. Selective and reduced integration is used for the shear strain energy, in order to avoid shear locking. 32 finite elements are used for discretization which leads to model with 65 nodes. The damage is considered to be in elements 21 and 22 which is between nodes 41 and 45 . The total number of DOF of the system is 195 .

Numerical experiments for detection and localization of the damage are performed on beams with clamped-clamped and clamped-free boundary conditions. Newmark's method is used for solving the ODE in time domain and Newton's method is used for solving the nonlinear system. Different speeds of rotation are assumed for the clamed-free boundary conditions.

First, a beam with clamped-clamped boundary conditions is analysed. An excitation frequency of 209 $\mathrm{rad} / \mathrm{s}$ is applied in all examples. Very often in real-world applications, like damage detection in wind turbine blades, the excitation frequency of the external conditions cannot be controlled, thus the methods for damage detection should work for wide variety of excitation frequencies. The selected excitation frequency is close to the fundamental frequency of the clampedclamped beam. It is close to the second linear frequency of clamped-free beam, and when the beam rotates with speed of $100 \mathrm{rad} / \mathrm{s}$, the excitation frequency is between the first and the second natural frequencies of the rotating beam.
The results for damage indexes of a beam with clamped-clamed boundary conditions calculated according to formulas (7) and (9) are presented in Fig. 1 and 2, respectively. DI2 has similar behavior as DI3. It can be seen that the location of the damage is successfully detected.

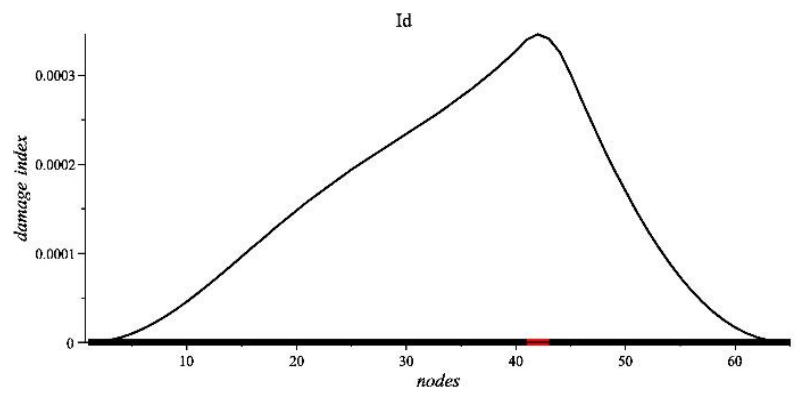

Fig. 1. Damage index $I^{\mathrm{d}}$ applied to beam with clampedclamped boundary conditions. The red segment presents damaged zone.

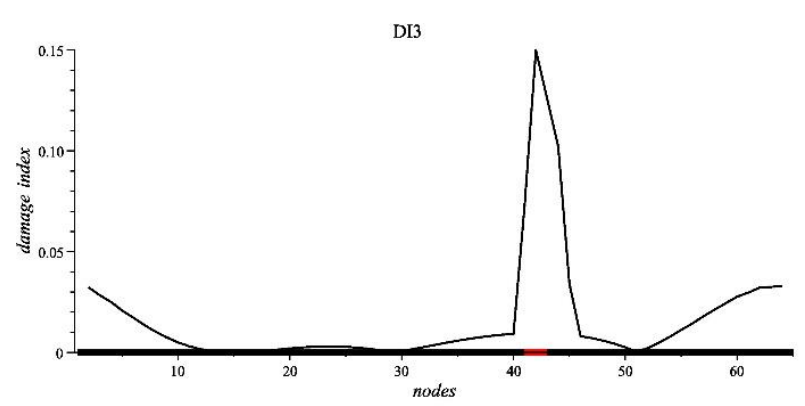

Fig. 2. Damage index DI3 applied to beam with clampedclamped boundary conditions. The red segment presents damaged zone.

When the boundary conditions of the beam are clamped-free, the presented formulas do not always localize the damage. The formulas have higher amplitudes on different location of the beam, depending on the shape of vibration. Fig. 3 and 4 present damage index DI3 for non-rotating and rotating beams.

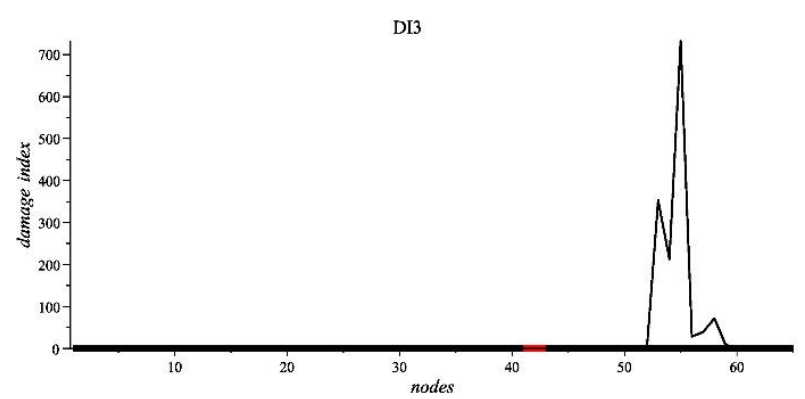

Fig. 3. Damage index DI3 applied to non-rotating beam with clamped-free boundary conditions.

In both cases, the damage is not appropriately localized. The reason for not localizing the damage is the free boundary condition. This boundary condition allows displacements of the beam with higher amplitude not only near the damaged area, but also near the free end and in other areas along the length, depending on the 
excitation frequency and the shape of vibration. The shapes of vibration of damaged and undamaged beams and their curvatures are presented in Figs. 5-8.



Fig. 4. Damage index DI3 applied to rotating beam with speed $100 \mathrm{rad} / \mathrm{s}$ with clamped-free boundary conditions.

The first two figures present the transverse displacements and the curvature of non-rotating clamped-free beam and the other two figures present the displacements and curvatures of rotating beams. It can be seen that the differences between damaged and undamaged beams are significant on locations different from the damaged area.

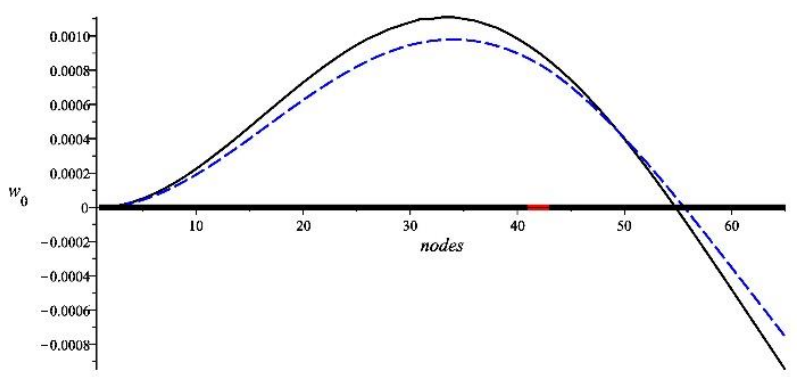

Fig. 5. Comparison of transverse displacement $w_{0}$ of damaged and undamaged beam at time $t=4.962 \mathrm{~s}$. Non-rotating beam with clamped-free boundary conditions. Blue dash line presents damaged beam.

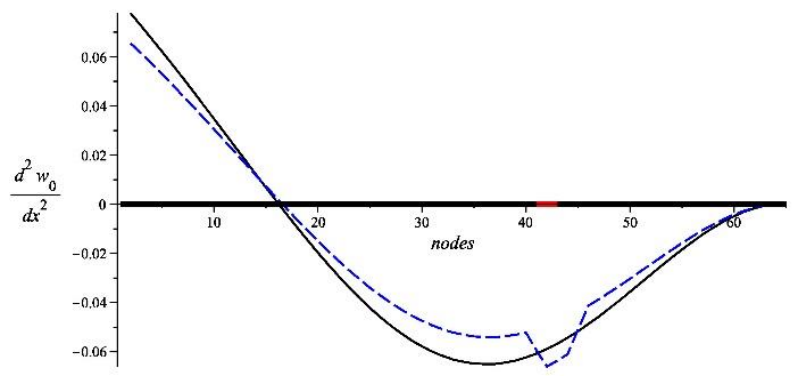

Fig. 6. Comparison of second derivative of transverse displacement $w_{0}$ of damaged and undamaged beam at time $t=$ 4.962 s. Non-rotating beam with clamped-free boundary conditions. Blue dash line presents damaged beam.

There are several possibilities for implementation of the selection criteria. For example, the selection criteria could be applied to the nodes which have higher amplitude in the curvature of the damaged beam. This criterion gives very good results in the numerical experiments, shown in Fig. 9. Further numerical experiments and improvements of the selection criteria will be developed in the future work of the authors



Fig. 7. Comparison of transverse displacement $w_{0}$ of damaged and undamaged beam at time $t=1.2 \mathrm{~s}$. Rotating beam with speed $100 \mathrm{rad} / \mathrm{s}$ with clamped-free boundary conditions. Blue dash line presents damaged beam.

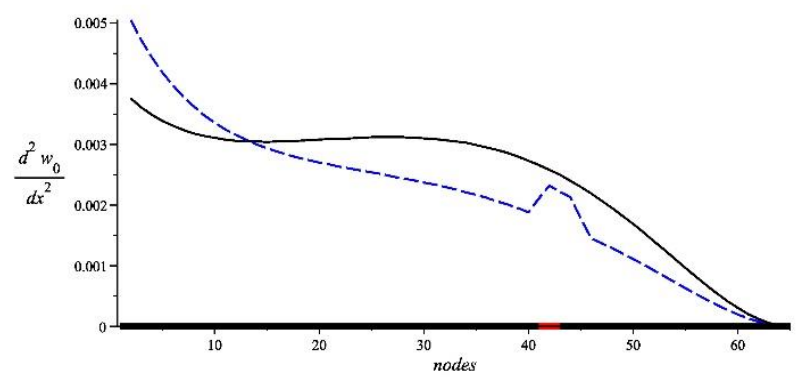

Fig. 8. Comparison of second derivative of transverse displacement $w_{0}$ of damaged and undamaged beam at time $t=$ $1.2 \mathrm{~s}$. Rotating beam with speed $100 \mathrm{rad} / \mathrm{s}$ with clamped-free boundary conditions. Blue dash line presents damaged beam.



Fig. 9. Damage index obtained by selection of second order derivatives applied to rotating beam with speed $100 \mathrm{rad} / \mathrm{s}$ with clamped-free boundary conditions.

\section{Conclusions}

The equation of motion for rotating beams that vibrate in one plane was derived and presented. Numerical experiments for localization of damages were performed by using vibration based methods and Poincaré maps. The influence of the speed of rotation on the shapes of vibration was outlined. It was shown that in the case of clamped-free boundary conditions, the methods not always localize correctly the damage. Additional methods which used appropriate selection of nodes were developed and it is shown that they perform much better. The future work will continue with the development and improvement of criteria for selection of the nodes and analysis of clamped-free beams with damages. 


\section{Acknowledgements}

The support of this work through the Bulgarian NSF Grant DNT-SO14 is gratefully acknowledged.

\section{References}

1. Trendafilova, I., Manoach, E., Nonlinear dynamics time series methods for fault detection and identification in structural and robotic systems. Book chapter in a book "Fault diagnosis in robotic and industrial systems", (G. G. Rigatos -Editor) iConceptPress, Queensland, Australia, I, 111-138, 2012.

2. García D., Cherniak, T., Trendafilova I. J. Phys.: Conf. Ser., 2015 , 628, 012086

3. Trendafilova I., Manoach E. Mech. Syst. Signal Pr., 22, 1092-1106 (2008).

4. Manoach E., Trendafilova I.. J.Sound Vib., 315 (3), 591-606 (2008)

5. Manoach, E., Samborski, S., Mitura, A., Warminski J.. . Int. J. Mech. Sci.; 62, 120-132 (2012).

6. Shabana, A. Dynamics of multibody systems, Cambridge University Press, (2013).

7. E. Manoach, J. Warminski, L. Kloda, A. Teter, Compos. Struct. 170, 26-39 (2017). 